\section{(1)}

CrossMark

\title{
International consensus guideline for reporting transmission electron microscopy results in the diagnosis of primary ciliary dyskinesia (BEAT PCD TEM Criterial
}

\author{
Amelia Shoemark (10 ${ }^{1,2}$, Mieke Boon ${ }^{3}$, Christoph Brochhausen ${ }^{4}$, \\ Zuzanna Bukowy-Bieryllo $\mathbb{1}^{5}$, Maria M. De Santi ${ }^{6}$, Patricia Goggin ${ }^{7}$, \\ Paul Griffin ${ }^{1,8}$, Richard G. Hegele ${ }^{9}$, Robert A. Hirst ${ }^{10}$, Margaret W. Leigh ${ }^{11}$, \\ Alison Lupton ${ }^{12}$, Karen MacKenney ${ }^{13}$, Heymut Omran ${ }^{14}$, Jean-Claude Pache ${ }^{15}$, \\ Andreia Pinto (16) ${ }^{16}$, Finn P. Reinholt ${ }^{17}$, Josep Schroeder ${ }^{4}$, Panayotis Yiallouros ${ }^{18}$ \\ and Estelle Escudier ${ }^{19}$, representing the BEAT-PCD Network Guideline \\ Development Group ${ }^{1}$
}

@ERSpublications

This tested and validated consensus statement provides clear terminology and defect definitions for the ultrastructural diagnosis of primary ciliary dyskinesia (PCD). The guideline will facilitate standardisation of PCD research and diagnosis. http://bit.ly/2RDkz7k

Cite this article as: Shoemark A, Boon M, Brochhausen C, et al. International consensus guideline for reporting transmission electron microscopy results in the diagnosis of primary ciliary dyskinesia (BEAT PCD TEM Criteria). Eur Respir J 2020; 55: 1900725 [https://doi.org/10.1183/13993003.00725-2019].

ABSTRACT Primary ciliary dyskinesia (PCD) is a heterogeneous genetic condition. European and North American diagnostic guidelines recommend transmission electron microscopy (TEM) as one of a combination of tests to confirm a diagnosis. However, there is no definition of what constitutes a defect or consensus on reporting terminology. The aim of this project was to provide an internationally agreed ultrastructural classification for PCD diagnosis by TEM.

A consensus guideline was developed by PCD electron microscopy experts representing 18 centres in 14 countries. An initial meeting and discussion were followed by a Delphi consensus process. The agreed guideline was then tested, modified and retested through exchange of samples and electron micrographs between the 18 diagnostic centres.

The final guideline a) provides agreed terminology and a definition of Class 1 defects which are diagnostic for PCD; b) identifies Class 2 defects which can indicate a diagnosis of PCD in combination with other supporting evidence; c) describes features which should be included in a ciliary ultrastructure report to assist multidisciplinary diagnosis of PCD; and d) defines adequacy of a diagnostic sample.

This tested and externally validated statement provides a clear guideline for the diagnosis of PCD by TEM which can be used to standardise diagnosis internationally.

This article has supplementary material available from erj.ersjournals.com

Received: 10 April 2019 | Accepted after revision: 24 Jan 2020

Copyright OERS 2020 


\section{Introduction}

Primary ciliary dyskinesia (PCD) is a heterogeneous inherited condition affecting 1:10000 of the population [1]. Symptoms usually begin early in life and include chronic nasal discharge and wet cough, progressing in childhood to recurrent upper and lower airway infections and eventual bronchiectasis [1]. As cilia are also present at the embryonic node, defects in nodal cilia may cause abnormalities of left-right laterality determination (situs abnormalities).

Diagnosis of PCD usually relies on a combination of tests because a single test cannot reliably diagnose all PCD types. These tests may include nasal nitric oxide measurement [2], assessment of ciliary waveform by high speed video microscopy [3], immunofluorescence (IF) analysis of ciliary proteins [4], transmission electron microscopy (TEM) and genetic testing. European and North American diagnostic guidelines differ in recommendations for diagnostic testing, although both agree that assessment of ciliary ultrastructure by TEM and/or bi-allelic mutations in a known PCD gene definitively confirm a PCD diagnosis [5-7]. However, there has been considerable heterogeneity among pathology reports describing PCD and in interpreting what constitutes a diagnostic defect. Defects of the ciliary axoneme can occur secondary to infection or inflammation of the airway mucosa, or in samples which have not been adequately prepared. Differences in reporting and interpretation of findings can lead to misdiagnosis.

In recent years, advances in genetic testing and molecular biology have redefined many aspects of PCD, whilst improving understanding of the basic science has further complicated the use of traditional electron microscopy terminology. For example the use of the term "radial spoke defect" has been used to describe both central complex defects such as those caused by RSPH4A mutations and microtubular disarrangement defects caused by CCDC39 mutations [8-11]. In other clinical entities, such as renal allograft pathology, an internationally agreed pathology classification known as the "Banff criteria" has been shown to significantly improve diagnostic precision, reproducibility and disease outcome [12, 13]. The aim of the present project was to provide an internationally agreed ultrastructural classification for the diagnosis of PCD by defining defects diagnostic for PCD, describing features which should be included in a report to assist multidisciplinary diagnosis of PCD and defining adequacy of a diagnostic sample.

\section{Methods \\ Guideline development}

The guideline development process is shown in figure 1. A group of electron microscopy experts representing 18 centres in 14 countries (Australia, Belgium, Canada, Cyprus, France, Germany, Italy, Norway, Poland, Portugal, Spain, Switzerland, the United Kingdom and the United States) met in person and via video link to develop a consensus statement for reporting ciliary ultrastructure by TEM at a COST Action BEATPCD meeting. Minutes from this meeting were used to create common themes and identify items for a Delphi consensus survey. The Delphi consensus survey consisted of three rounds with aims to: 1) define defects diagnostic for PCD; 2) describe features which should be included in a report to assist multidisciplinary diagnosis of PCD; and 3) define adequacy of a diagnostic sample. Consensus was considered to be achieved if more than $80 \%$ of the participants agreed. Consultation with the wider BEATPCD community was conducted at the next BEATPCD meeting.

\section{Guideline validation}

Once a draft guideline was complete a series of TEM sections were distributed among the group and the guideline was tested and modified for inconsistencies and clarity. A list of 16 cases representing patients who had a genetically confirmed diagnosis of PCD or who conclusively did not have PCD were used for the guideline validation. Four centres were assigned four cases from the following to prepare: CCDC114,

Affiliations: ${ }^{1}$ Royal Brompton Hospital, London, UK. ${ }^{2}$ School of Medicine, University of Dundee, Dundee, UK. ${ }^{3}$ Dept of Pediatrics, University Hospital Leuven, Leuven, Belgium. ${ }^{4}$ Institute of Pathology, University Regensburg, Regensberg, Germany. Institute of Human Genetics, Polish Academy of Sciences, Poznan, Poland. ${ }^{6}$ Dept of Pathology, University Hospital of Siena, Siena, Italy. ${ }^{7}$ University Hospital Southampton NHS Foundation Trust, Southampton, UK. ${ }^{8}$ Royal Childrens Hospital, Melbourne, Australia. ${ }^{9}$ Hospital for Sick Children-University of Toronto, Toronto, ON, Canada. ${ }^{10}$ Dept of Respiratory Sciences, University of Leicester, Leicester, UK. ${ }^{11}$ Dept of Pediatrics and Marsico Lung Institute, University of North Carolina, Chapel Hill, NC, USA. ${ }^{12}$ Pathology Dept, Greater Glasgow and Clyde, Queen Elizabeth University Hospital, Glasgow, UK. ${ }^{13} \mathrm{NSW}$ Health Pathology, Concord Repatriation General Hospital, Sydney, Australia. ${ }^{14}$ Dept of Pediatrics, University Hospital Muenster, Muenster, Germany. ${ }^{15}$ University Hospital of Geneva, Geneva, Switzerland. ${ }^{16}$ Instituto de Medicina Molecular, Lisbon, Portugal. ${ }^{17}$ Oslo University Hospital, Oslo, Norway. ${ }^{18}$ Medical School, University of Cyprus, Nicosia, Cyprus. ${ }^{19}$ Sorbonne Université, Faculté de Médecine, INSERM UMR_S933, (APHP) Assistance Publique Hôpitaux de Paris and CHIC (Centre Hospitalier Intercommunal de Créteil), Paris, France.

Correspondence: Amelia Shoemark, Primary Ciliary Dyskinesia Centre, Paediatric Respiratory Medicine, Royal Brompton Hospital, Sydney Street, London, SW3 6NP, UK. E-mail: a.shoemarkadundee.ac.uk 
FIGURE 1 Flow chart outlining the methodology for development of the transmission electron microscopy (TEM) consensus guideline. Results of Delphi surveys and case reviews can be found in the supplementary

Kick off meeting at BEATPCD conference in Paris to identify opinions and items to include in the survey

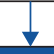

Initial Delphi consensus survey

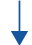

Consultation and discussion with wider PCD community at satellite meeting during the ERS conference

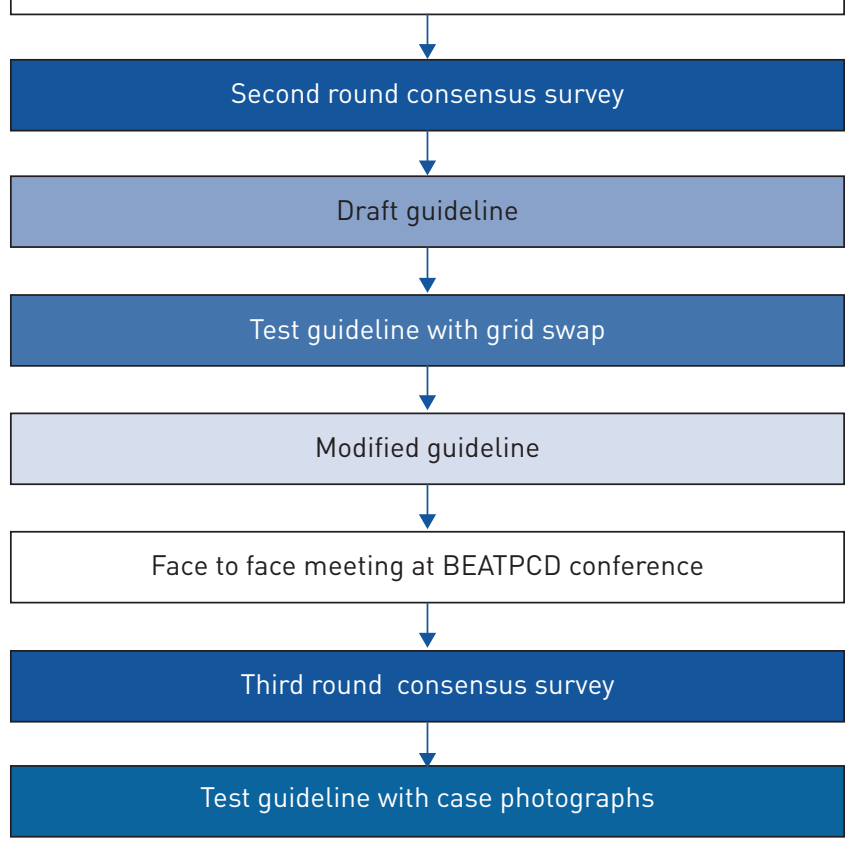
information.

CCDC39, CCDC40, CCNO, DNAAF1, DNAAF3, DNAH5, DNAH11, DRC1, RSPH4A, cystic fibrosis (CF), healthy volunteer $(\times 2)$, inadequate sample $(\times 2)$ and unaffected sibling of a patient with PCD. Centres at Southampton (UK), Toronto (Canada), Paris (France) and Muenster (Germany) sectioned and stained four grids from four cases each. These grids were sent to London where they were quality checked, anonymised, randomised and distributed by an individual who was not part of the consensus group. Seventeen centres then received four grids and one centre received images (centres did not receive their own grids).

All 17 centres assessed the four grids, each centre using its own technology for making and interpreting images, while the 18th centre received images. The centres completed a report form based on the consensus statement and returned representative images. The form included class of defect, ultrastructural defect, adequacy of the sample and a summary of key findings. One centre reviewed images taken at the distribution centre in London, rather than grids, due to technical difficulties.

Results of the validation exercise were discussed in a face to face meeting of the group. One additional online survey was distributed to clarify points from this meeting and the guideline was modified accordingly. Results of the guideline surveys and validation can be found in the supplementary material.

Finally images from the cases with CCDC39, DNAAF3, DNAH5, RSPH4A and CF were used to reassess the modified completed guideline. This validation exercise was completed by all consensus statement authors and six additional electron microscopists who were not directly involved in developing the consensus statement.

\section{Results}

This guideline describes assessment and reporting of ciliated epithelial samples fixed in glutaraldehyde, dehydrated, embedded in resin and stained with heavy metals (lead citrate and uranyl acetate or equivalent).

\section{Normal ultrastructure of respiratory cilia}

The consensus group identified that it is crucial for the observer to be familiar with the appearance of normal ciliary ultrastructure and PCD defects from extensive samples, using local sampling, processing 
and visualisation methods, before embarking on diagnostic testing for PCD. Figure 2 shows the normal ultrastructure of a healthy respiratory cilium. The axoneme of motile cilia is composed of the well-known $9+2$ structure, where nine peripheral microtubule doublets surround the central pair of single microtubules. Each microtubular doublet is connected with the central pair by radial spokes and neighbouring microtubular doublets are connected by the nexin dynein regulatory complex (N-DRC). The outer microtubular doublets contain regularly repeating hook shaped structures known as outer dynein arms (ODAs) and inner dynein arms (IDAs), which are responsible for the generation of ciliary motion through ATPase activity. Essential units repeat along the axoneme approximately every $96 \mathrm{~nm}$. Each doublet contains, in a $96 \mathrm{~nm}$ repetition, four double headed ODAs, six different single-headed IDAs and one double-headed IDA isoform, one N-DRC and three radial spokes [14-17]. Differences in the repetition of these units and of their density results in differing contrast on TEM. The electron dense ODA is easier to visualise in a 70-100 nm TEM section than the less dense IDA and N-DRC because more ODA dynein heavy chains are present within the slice. Ultrastructure at the tip and base of the cilium is different and this should be considered when assessing for PCD defects.

Class 1 and Class 2 defects, terminology, adequacy of sampling and consensus definitions for the diagnosis of PCD by TEM

The expert consensus group identified two classes of PCD TEM diagnostic defects: Class 1 defects, which are considered "hallmark" defects confirming a diagnosis in a patient with symptoms of the condition; and Class 2 defects, which indicate a diagnosis of PCD in a patient with clinical symptoms of the condition if consistent across more than one sample or after cell culture AND if consistent with other test results. The two classes of defects are shown in table 1 and are defined in the text. The group decided to distinguish between the two classes of defect because Class 1 defects are diagnostic whereas Class 2 defects can be more difficult to recognise and can be similar to secondary defects.

\section{Class 1 defects}

Class 1 defects are diagnostic of PCD in a patient with clinical symptoms of the condition. Class 1 defects are confirmed following assessment of more than 50 axonemes in transverse section from several cells. Care should be taken to assess cross sections from both proximal (in the region of the microvili) and distal regions of the axoneme. Dynein arms should be assessed in cross sections with clear structural features and an intact ciliary membrane. Microtubular arrangement may be assessed in a larger number of cross sections with an intact membrane in which the dynein arms may not be clear. Examples of Class 1 defects are shown in figure 3.
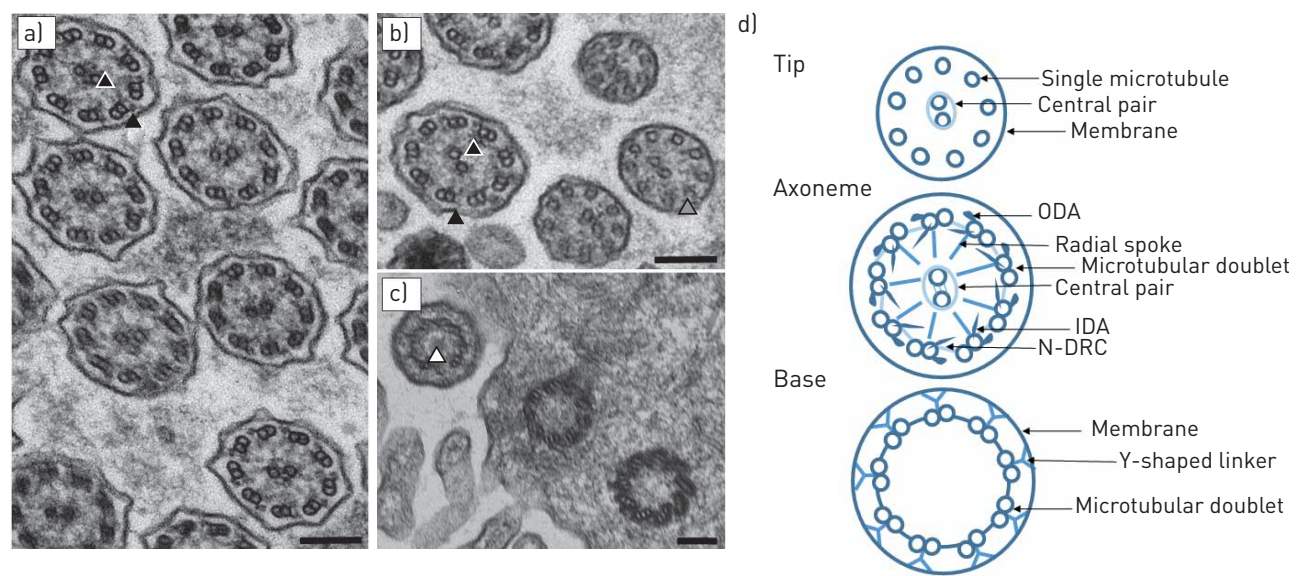

FIGURE 2 Electron micrographs showing normal ciliary ultrastructure in cross section at a) the core of the ciliary axoneme with a 9+2 microtubular arrangement; b) the tip of the cilium with single microtubules; and c) the base of the axoneme. Panel d) gives a diagrammatic representation of ciliary cross sections at the tip, central axoneme and base for normal ciliary ultrastructure. The black arrowheads depict the outer dynein arms (ODAs) and the black arrowheads with a white outline depict the inner dynein arms (IDAs). Grey arrowheads indicate the tip of the cilium with single microtubules/these are a normal part of ciliary ultrastructure and should not be assessed for diagnosis of Class 1 or Class 2 defects). White arrowheads indicate where there is no central pair. Microtubular doubles are often linked to the ciliary membrane with Y-shaped linker. N-DRC: nexin dynein regulatory complex. 
TABLE 1 Summary of Class 1 and Class 2 defects for the ultrastructural diagnosis of primary ciliary dyskinesia (PCD)

\author{
Class 1 defects: hallmark diagnostic defects \\ ODA defect \\ ODA and IDA defect \\ Microtubular disorganisation and IDA defect \\ Class 2 defects: indicate a PCD diagnosis with other supporting evidence \\ Central complex defect \\ Mislocalisation of basal bodies with few or no cilia \\ Microtubular disorganisation with IDA present \\ ODA absence from $25-50 \%$ of cross sections \\ Combined IDA and ODA absence from $25-50 \%$ of cross sections
}

ODA: outer dynein arm; IDA: inner dynein arm.

\title{
ODA defects
}

Absence of the whole or a larger part of the ODA structure from the majority (five or more) of microtubular doublets in the majority (more than 50\%) of cilia cross sections. The defects arise from mutations in genes coding for ODA structural proteins (e.g. DNAH5) and ODA docking proteins (e.g. CCDC151). IF analysis usually shows an absence of the outer dynein heavy chain protein DNAH5.

\section{ODA and IDA defects}

Absence of the whole or a larger part of the ODA structure from the majority (five or more) of microtubular doublets in the majority (more than 50\%) of cilia cross sections AND absence of the whole

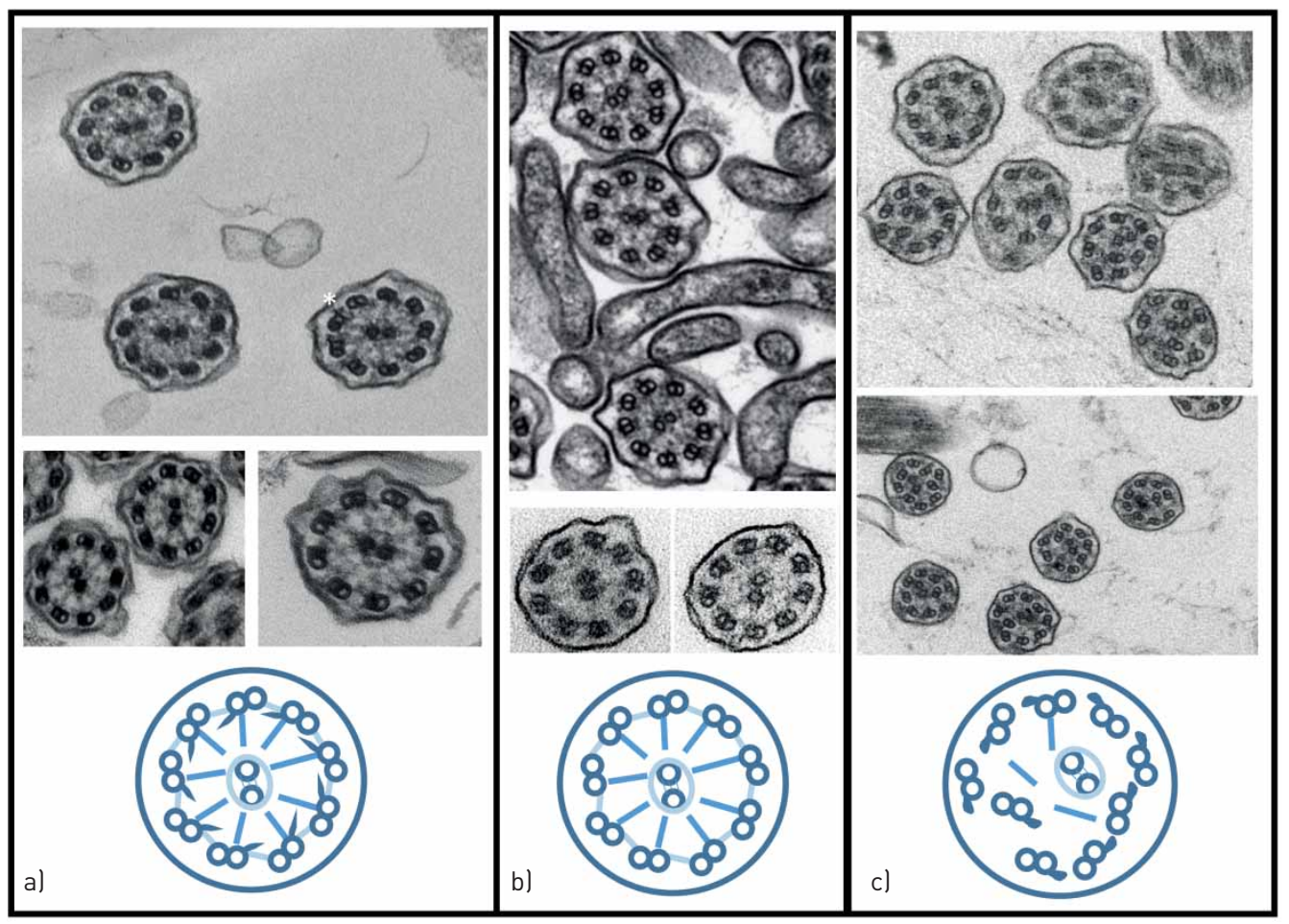

FIGURE 3 Electron micrographs and diagrams of Class 1 ultrastructural defects in cross section. a) Outer dynein arm (ODA) defect; b) ODA and IDA defect; c) microtubular disorganisation and IDA defect. It should be noted that not all arms are completely missing. Although the curved hook structure of the ODA is not present in its entirety the white star in al indicates some remaining proximal structure likely to be the docking complex. Class 1 defects are diagnostic of primary ciliary dyskinesia (PCD) in patients with symptoms of the condition. Class 1 defects are confirmed following assessment of more than 50 axonemes in transverse section. Dynein arms should be assessed in cross sections with clear structural features and an intact ciliary membrane as depicted. Microtubular arrangement may be assessed in a larger number of cross sections with an intact membrane in which the dynein arms may not be clear. 
or a larger part of the IDA structure from the majority (seven or more) of microtubular doublets in the majority (more than 50\%) of cilia cross sections. If the number of IDAs cannot be accurately determined, samples should be reported as "ODA defect (+/- IDA defect)". These defects often arise from mutations in dynein assembly genes. IF analysis shows the absence of proteins such as DNAH5 and DNALI1.

\section{Microtubular disorganisation and IDA defects}

Disruption of the $9+2$ symmetry of the microtubules in more than $25 \%$ of cross sections combined with the absence of the whole or a larger part of the IDA structure from the majority (more than seven) of microtubular doublets in the majority (more than 50\%) of cilia axonemes visualised in cross section. The defects almost always arise from CCDC39 or CCDC40 mutations [7]. IF analysis in these cases shows an absence of N-DRC proteins such as GAS8 and IDA proteins such as DNALI1.

\section{Class 2 defects}

Class 2 defects can indicate a diagnosis of PCD in a patient with clinical symptoms of the condition if consistent across more than one sample AND if consistent with other results (such as those from IF, high speed video microscopy or genetic analysis). They may require assessment of more ciliary axonemes than Class 1 defects. Care should be taken to assess cross sections from several cells and both proximal (in the region of the microvili) and distal regions of the axoneme. Dynein arms should be assessed in cross sections with clear structural features and an intact ciliary membrane. Microtubular arrangement may be assessed in a larger number of cross sections with an intact membrane in which the dynein arms may not be clear. Examples of Class 2 defects are shown in figure 4.

\section{Central complex defects}

Central complex defects consist of a proportion of normal cross sections and consistent cross sections with one or both of the central microtubules absent (usually more than 20\%). Occasional double central pairs or translocation of the outer microtubule to the central region (the " $8+1$ pattern") may be seen. Translocation typically occurs approaching the ciliary tip. In longitudinal sections, intermittent or complete loss of the central pair or transposition of an outer doublet may also be seen. Some microtubular disorganisation is often seen. These TEM findings are reported to be associated with genetic defects of the radial spoke components (e.g. RSPH4A, RSPH1, RSPH9 and DNAJB13) [18-20], which can be confirmed by IF for radial spoke head proteins. In some cases high speed video microscopy can show circling of cilia when observed from above.

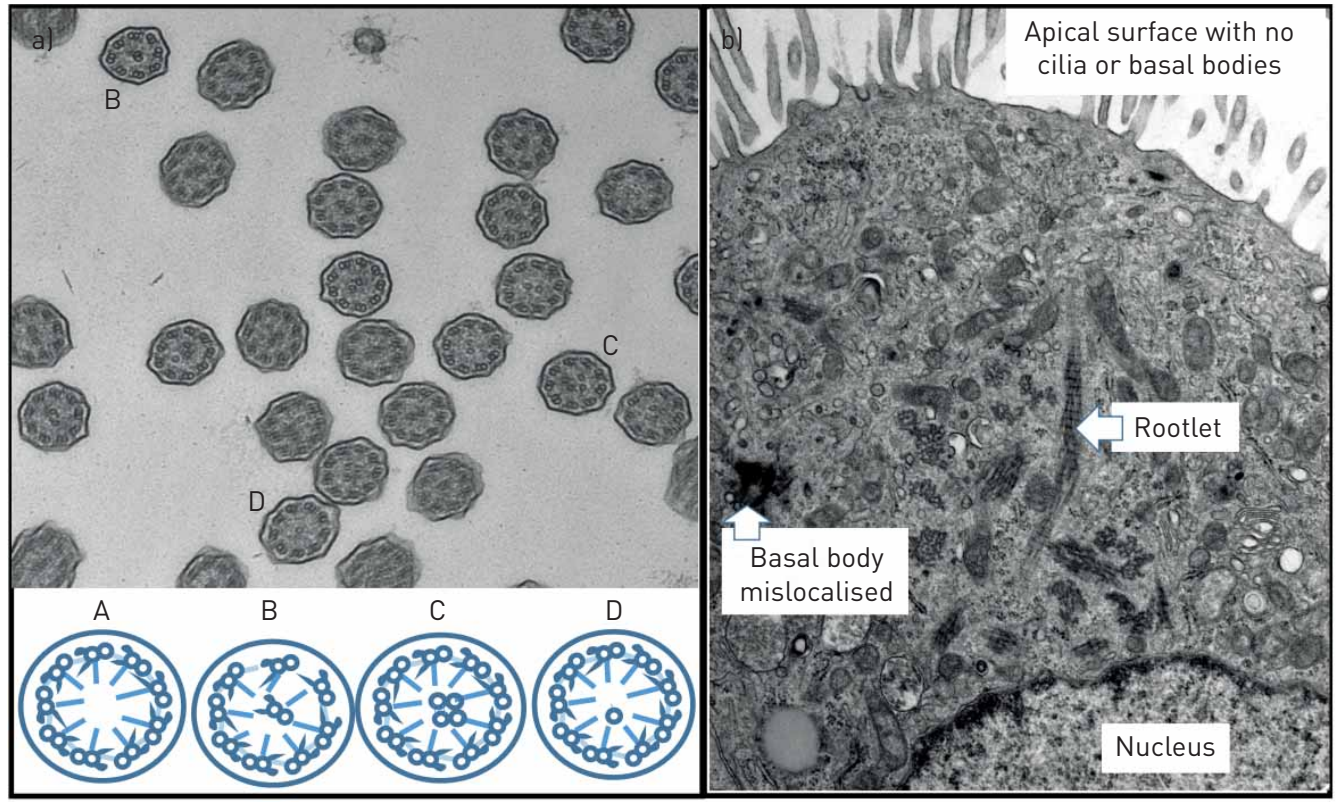

FIGURE 4 Electron micrographs of Class 2 ultrastructural defects. a) Central complex defect with features demonstrating: (A) a lack of a central pair; (B) a transposed outer microtubular doublet; (C) a double central pair; and (D) a single central tubule. The micrograph was taken from an individual with an RSPH4A mutation. b) Mislocalisation of basal bodies with few or no cilia from an individual with a CCNO mutation. 
Mislocalisation of basal bodies with few or no cilia

Findings are typically of no or very few ciliary cross sections in combination with failure of the majority of basal bodies to dock at the apical surface of the cell, meaning they are consistently seen within the cytoplasm. These findings are associated with genetic variants in CCNO and MCIDAS and only a few cilia are seen per cell by high speed video microscopy $[21,22]$. The genetic defects result in reduced generation of multiple motile cilia. In the rare cilia that are visualised, the ultrastructure of the axoneme in CCNO cases can be normal but in individuals with MCIDAS mutations it can lack ODAs.

\section{Microtubular disorganisation}

Microtubular disorganisation is indicated by consistent disruption of the $9+2$ symmetry of the microtubules in cross sections throughout otherwise healthy samples. The absence of the N-DRC link is sometimes noted; however, the majority of cross sections usually have normal ultrastructure with ODAs and IDAs present. These findings might be associated with mutations in CCDC65, DRC1 and GAS8 encoding N-DRC proteins [23-25]. Subtle beat pattern abnormalities including fast or disrupted beat pattern and an absence of N-DRC proteins such as GAS8 are observed by IF in these cases. The IDA is present in more than $50 \%$ of cross sections, distinguishing this case from the Class 1 microtubular disorganisation and IDA defect.

\section{ODA absence from $25-50 \%$ of cross sections}

This definition is the same as for a Class 1 "ODA defect", except for the dynein arm(s) being absent in a minority of cilia and numerous normal ciliary cross sections being present. A pattern to the defect may be observed (e.g. for individuals with recessive DNAH9 mutations the distal ciliary axonemes lack ODAs whereas the proximal ciliary axonemes are normal) [26,27]. Where a pattern to the defect is observed by TEM, IF may also show a partial absence of DNAH5.

\section{Combined IDA and ODA absence from $25-50 \%$ of cross sections}

This definition is the same as for a Class 1 "ODA and IDA defect", except for the dynein arms being absent in a minority of cilia and numerous normal ciliary cross sections being present. Some static cilia may be present by high speed video microscopy and IF may or may not show a partial absence of DNAH5 and DNALI1. This is seen, for example, in patients with the missense mutation His154Pro in CCDC103 [28, 29].

\section{The transmission electron microscopy cilia report}

A TEM report should conform to general considerations for good practice in pathology reporting, as follows: 1) reports should be clear and accurate and the overall result or conclusion must be clearly visible; 2) patients must be identified on reports by at least two unique items of information (e.g. full name and date of birth); 3) it must be stated if the testing is incomplete and/or where the minimum quality is not achieved; and 4) for PCD testing it should be made clear to the person receiving the report that normal ultrastructure does not exclude a diagnosis. Items agreed by the expert group as essential and desirable for an electron microscopy report in the diagnosis of PCD are described in table 2.

TABLE 2 Items to include in a transmission electron microscopy (TEM) report for the diagnosis of primary ciliary dyskinesia (PCD)

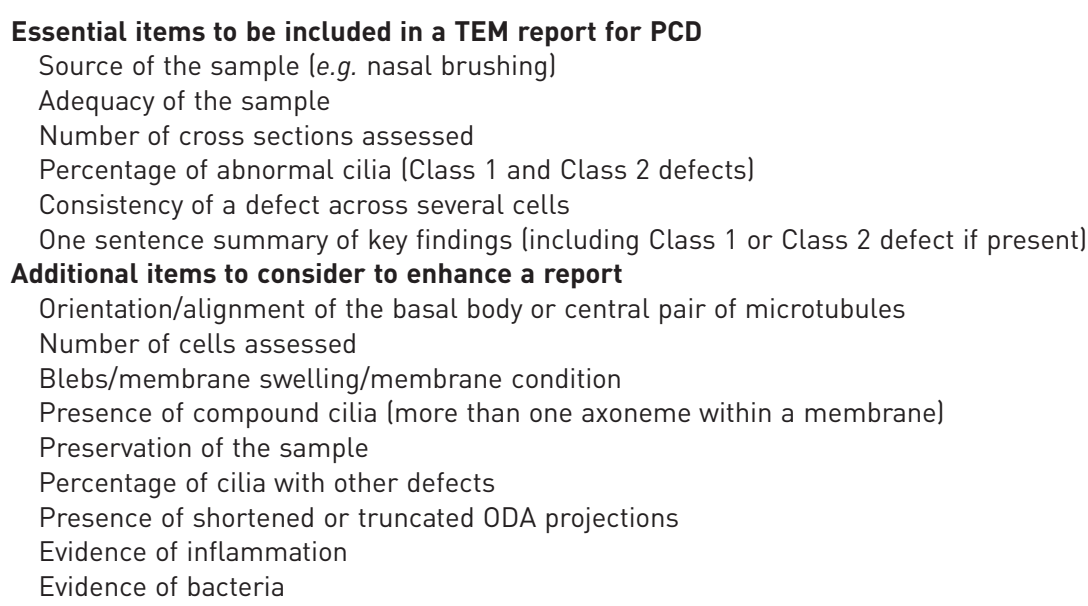


Summary of guideline development survey results

In round one, consensus was obtained on the three hallmark defects shown in table 1 and participants suggested definitions. More than $80 \%$ agreed that the following were Class 2 defects: central complex defects, mislocalisation of basal bodies with few or no cilia, ODA absence from $25-50 \%$ of cross sections, and combined IDA and ODA absence from $25-50 \%$ of cross sections. Microtubular disorganisation was added to this list following the validation exercise. Items to include in a report were also agreed (table 2). The group did not reach consensus on the following items as being Class 1 or Class 2 defects and inclusion or exclusion of these should be at the discretion of the observer as evidence becomes available: isolated IDA defects [30], ODA loss from less than $25 \%$ of cross sections, ODA and IDA loss from less than $25 \%$ of cross sections, orientation defects [31-33] and ciliary length defects. Future research should focus on the relevance of these defects and the guideline should be updated accordingly. In rounds two and three, definitions of hallmark defects were agreed along with the criteria for sample adequacy (i.e. more than 50 cross sections with intact ciliary membranes). Full details of the guideline development process are given in the supplementary material.

\section{Summary of guideline validation results}

Two rounds of validation were conducted and there was 100\% participation in this activity (across all 18 centres). In round one there were no false positive diagnoses (i.e. no non-PCD sample was classed as having a Class 1 hallmark PCD defect). Seventeen out of 68 sections (25\%) were described as insufficient or inadequate for assessment. These included $100 \%$ returns on a CCNO case and $75 \%$ returns for a sample included as an inadequate sample. Twenty-five out of 25 cases (100\%) were correctly identified as Class 1 hallmark defects; however, eight out of 25 returns (32\%) recorded an incorrect name for the Class 1 defect. Two cases were described as having a Class 1 defect when they had a Class 2 defect or normal ultrastructure. Five out of six cases were correctly identified as Class 2 defects. Four normal ultrastructure cases were erroneously identified as Class 2 defects.

The guideline was modified and in round two there were no false positive diagnoses (i.e. no non-PCD sample was classed as having a Class 1 hallmark PCD defect) and normal ultrastructure was identified by 18 out of 18 centres. There were no false negatives and there was $100 \%$ correct identification of a Class 1 hallmark defect. These included 18 out of 18 correctly identified microtubular disorganisation+IDA defects, 17 out of 18 ODA defects (one was judged as an ODA+IDA defect) and 17 out of 18 ODA+IDA defects (one was judged as an ODA defect). Furthermore, there was $100 \%$ correct identification of a Class 2 defect. Fifteen out of 18 correctly identified a central complex defect, whereas three judged this to be microtubular disorganisation (and thus stressing the importance of the additional evidence requirement for Class 2 defects). $94 \%$ of reviewers gave a correct ultrastructural defect according to the genetic diagnosis. Agreement between observers $(\kappa)$ was $0.76-1.0$ (where 0: no agreement and 1: complete agreement). For external validation a further six electron microscopists were given the guideline to read and then completed the image validation. All six observers identified the correct ultrastructural defects for all cases.

\section{Discussion}

Next generation sequencing and molecular advances have resulted in fast-paced improvement of PCD diagnosis over recent years. TEM is valuable in asserting and/or confirming a diagnosis of PCD, and as a guide for genetic testing, as there is a clear link between ultrastructural defects and the group of affected genes. This is useful especially when variants of unknown significance are identified. Pathogenic mutations in known PCD genes currently account for only $65-75 \%$ of cases of PCD confirmed by TEM. A table of known TEM phenotypes according to genotype is available in the supplementary material. We present the first internationally agreed guideline for reporting of ciliary biopsies for the diagnosis of PCD. This guideline is designed to be a flexible formulation, it will require updating and evolution as new evidence becomes available. These recommendations are minimum requirements and professional judgement and experience is of paramount importance in many circumstances.

\section{PCD with normal ultrastructure}

It has been shown repeatedly that the diagnostic accuracy of TEM is not sufficient to exclude a diagnosis, as up to $30 \%$ of all PCD cases are described as having normal or near normal ciliary ultrastructure [34-36]. This includes defects in the large dynein heavy chain, DNAH11, which accounts for a substantial number of cases $[37,38]$. In another group of patients, subtle changes to ultrastructure can be seen but alone these are non-diagnostic (e.g. defects in HYDIN which affect the c2b projection of the central pair) [39]. The current statement introduces the concept of Class 2 defects to address limitations with the identification of subtle changes. Class 2 defects support a diagnosis but should not be used without other diagnostic tests. Many of these Class 2 defect changes are secondary to the causative molecular defect (e.g. radial spoke head gene 
defects result in secondary loss of the central pair) [19]. It must be stressed that some Class 2 defects, if not confirmed in a second sample or after cell culture, may also be the result of non-PCD related causes.

\section{Limitations of electron microscopy in the diagnosis of PCD}

Initial validation of the guideline revealed that smaller, inadequate samples were most likely to lead to a misdiagnosis (see supplementary material). It was also clear that individual processing techniques and familiarity with the local appearance of cilia was paramount for interpretation. Due to differences in equipment availability and local procedures it is not currently possible to standardise all methodology in sample processing and visualisation. In the future, a consensus methodology may be developed; however, we have addressed methodological differences by recommending that extensive normal and PCD samples are visualised using local techniques before a diagnostic sample is interpreted.

Secondary ultrastructural defects in cilia can be caused by acute or chronic respiratory infections, inflammatory respiratory disease (i.e. asthma), environmental and demographic factors (smoking, pollution and age), or sample handling [40]. It is common to observe secondary defects in samples examined by TEM (e.g. microtubular defects, which have been observed in up to $10 \%$ of healthy controls) [41, 42]. The appearance of secondary defects can overlap with the appearance of positive PCD cases (e.g. microtubular disorganisation and central pair abnormalities). Misinterpretation of these defects can be prevented by taking care to avoid sampling when the patient is unwell, the use of cell culture from the respiratory epithelium (air-liquid interface or spheroid cultures) to remove conditions causing the secondary effects, consideration of the health of individual cells in the sample and by repeating the biopsy [43, 44]. To ensure that occasional secondary defects in the sample do not bias interpretation, we recommend assessment of at least 50 axonemes in transverse section from a number of different cells. We recommend that dynein arms should be assessed in cross sections with clear structural features and intact ciliary membranes. Microtubular arrangement may be assessed in a larger number of cross sections with intact membranes, in which the dynein arms are not clear, although unhealthy cells or compound cilia should be avoided. For Class 2 defects we recommend assessment of more than one sample or a following cell culture. Paramount is the consistency of a Class 2 defect with results from other tests, such as high speed video, IF analysis, nasal nitric oxide measurement and genotyping.

\section{Summary}

The performance of TEM for PCD diagnosis (i.e. sensitivity and specificity) is difficult to accurately assess when there are no agreed diagnostic criteria. This consensus statement is tested and validated and provides a clear guideline for the diagnosis of PCD by TEM, which can be used to standardise diagnosis internationally.

Acknowledgements: One representative lead author per centre (usually the individual assessing and reporting the TEM, and representing the opinions of each centre by completing the Delphi survey) has been included in the authorship of this report. However, several additional experts have attended meetings, commented on this consensus statement and contributed their expert opinion and technical skills during its development. We would like to thank: Sverre-Henning Brorson, Tom Burgoyne, Suzanne Crowley, Chris O'Callaghan, Mellisa Dixon, Regan Doherty, Catherine Faucon, Paul French, Raffaella Guazzo, Yew Meng Heng, Claire Hogg, Merete Helgesen, Louise Hughes, Martine Jaspers, Panayiotis Kouis, Kyriacou Kyriacos, Jane Lucas, Virginia Mancini, Paul Martinello, Rana Mitri, Elena Moretti, José Moura Nunes, Anton Page, Phil Robinson, Anna Rowinska, Andrew Rutman, Heiko Siegmund, Jennifer Sweeney, James Thompson and Valerie Vlaeminck. In addition, we are especially grateful to Farheen Daudvohra for anonymising and randomising sections and collating data for the validation of this guideline

Support statement: This project was conducted as part of BEAT-PCD, a network of multidisciplinary researchers and clinicians funded by EU COST Action (BM1407). Funding information for this article has been deposited with the Crossref Funder Registry.

Conflict of interest: A. Shoemark has nothing to disclose. M. Boon reports COST and STSM funding, during the conduct of the study; grants from Horizon 2020 MyCyFAPP, outside the submitted work. C. Brochhausen has nothing to disclose. Z. Bukowy-Bieryllo has nothing to disclose. M.M. De Santi has nothing to disclose. P. Goggin has nothing to disclose. P. Griffin has nothing to disclose. R.G. Hegele has nothing to disclose. R.A. Hirst has nothing to disclose. M.W. Leigh has nothing to disclose. A. Lupton has nothing to disclose. K. MacKenney has nothing to disclose. H. Omran has nothing to disclose. J-C. Pache has nothing to disclose. A. Pinto has nothing to disclose. F.P. Reinholt has nothing to disclose. J. Schroeder has nothing to disclose. P. Yiallouros has nothing to disclose. E. Escudier has nothing to disclose.

\section{References}

1 Lucas JS, Burgess A, Mitchison HM, et al. Diagnosis and management of primary ciliary dyskinesia. Arch Dis Child 2014; 99: 850-856.

2 Collins SA, Gove K, Walker W, et al. Nasal nitric oxide screening for primary ciliary dyskinesia: systematic review and meta-analysis. Eur Respir J 2014; 44: 1589-1599. 
3 Rubbo B, Shoemark A, Jackson CL, et al. Accuracy of high-speed video analysis to diagnose primary ciliary dyskinesia. Chest 2019; 155: 1008-1017.

4 Shoemark A, Frost E, Dixon M, et al. Accuracy of immunofluorescence in the diagnosis of primary ciliary dyskinesia. Am J Respir Crit Care Med 2017; 196: 94-101.

5 Shapiro AJ, Davis SD, Polineni D, et al. Diagnosis of primary ciliary dyskinesia. An official American Thoracic Society clinical practice guideline. Am J Respir Crit Care Med 2018; 197: e24-e39.

6 Lucas JS, Barbato A, Collins SA, et al. European Respiratory Society guidelines for the diagnosis of primary ciliary dyskinesia. Eur Respir J 2017; 49: 1601090.

7 Shoemark A, Dell S, Shapiro A, et al. ERS and ATS diagnostic guidelines for primary ciliary dyskinesia: similarities and differences in approach to diagnosis. Eur Respir J 2019; 54: 1901066.

8 Sturgess JM, Chao J, Turner JA. Transposition of ciliary microtubules: another cause of impaired ciliary motility. N Engl J Med 1980; 303: 318-322.

9 Sturgess JM, Chao J, Wong J, et al. Cilia with defective radial spokes: a cause of human respiratory disease. $N$ Engl J Med 1979; 300: 53-56.

10 Frommer A, Hjeij R, Loges NT, et al. Immunofluorescence analysis and diagnosis of primary ciliary dyskinesia with radial spoke defects. Am J Respir Cell Mol Biol 2015; 53: 563-573.

11 Antony D, Becker-Heck A, Zariwala MA, et al. Mutations in CCDC39 and CCDC40 are the major cause of primary ciliary dyskinesia with axonemal disorganization and absent inner dynein arms. Hum Mutat 2013; 34: 462-472.

12 Solez K, Axelsen RA, Benediktsson $\mathrm{H}$, et al. International standardization of criteria for the histologic diagnosis of renal allograft rejection: the Banff working classification of kidney transplant pathology. Kidney Int 1993; 44: 411-422.

13 Racusen LC, Solez K, Colvin RB, et al. The Banff 97 working classification of renal allograft pathology. Kidney Int 1999; 55: 713-723.

14 Kurkowiak M, Zietkiewicz E, Witt M. Recent advances in primary ciliary dyskinesia genetics. J Med Genet 2015; 52: 1-9.

15 Afzelius BA. A human syndrome caused by immotile cilia. Science 1976; 193: 317-319.

16 Burgoyne T, Dixon M, Luther $\mathrm{P}$, et al. Generation of a three-dimensional ultrastructural model of human respiratory cilia. Am J Respir Cell Mol Biol 2012; 47: 800-806.

17 Satir P, Christensen ST. Structure and function of mammalian cilia. Histochem Cell Biol 2008; 129: 687-693.

18 Onoufriadis A, Shoemark A, Schmidts M, et al. Targeted NGS gene panel identifies mutations in RSPH1 causing primary ciliary dyskinesia and a common mechanism for ciliary central pair agenesis due to radial spoke defects. Hum Mol Genet 2014; 23: 3362-3374.

19 Castleman VH, Romio L, Chodhari R, et al. Mutations in radial spoke head protein genes RSPH9 and RSPH4A cause primary ciliary dyskinesia with central-microtubular-pair abnormalities. Am J Hum Genet 2009; 84: 197-209.

20 El Khouri E, Thomas L, Jeanson L, et al. Mutations in DNAJB13, encoding an HSP40 family member, cause primary ciliary dyskinesia and male infertility. Am J Hum Genet 2016; 99: 489-500.

21 Boon M, Wallmeier J, Ma L, et al. MCIDAS mutations result in a mucociliary clearance disorder with reduced generation of multiple motile cilia. Nat Commun 2014; 5: 4418

22 Wallmeier J, Al-Mutairi DA, Chen CT, et al. Mutations in CCNO result in congenital mucociliary clearance disorder with reduced generation of multiple motile cilia. Nat Genet 2014; 46: 646-651.

23 Olbrich H, Cremers C, Loges NT, et al. Loss-of-function GAS8 mutations cause primary ciliary dyskinesia and disrupt the nexin-dynein regulatory complex. Am J Hum Genet 2015; 97: 546-554.

24 Horani A, Brody SL, Ferkol TW, et al. CCDC65 mutation causes primary ciliary dyskinesia with normal ultrastructure and hyperkinetic cilia. PLoS One 2013; 8: e72299.

25 Wirschell $\mathrm{M}$, Olbrich $\mathrm{H}$, Werner $\mathrm{C}$, et al. The nexin-dynein regulatory complex subunit DRC1 is essential for motile cilia function in algae and humans. Nat Genet 2013; 45: 262-268.

26 Fassad MR, Shoemark A, Legendre M, et al. Mutations in outer dynein arm heavy chain DNAH9 cause motile cilia defects and situs inversus. Am J Hum Genet 2018; 103: 984-994.

27 Loges NT, Antony D, Maver A, et al. Recessive DNAH9 loss-of-function mutations cause laterality defects and subtle respiratory ciliary-beating defects. Am J Hum Genet 2018; 103: 995-1008.

28 Shoemark A, Moya E, Hirst RA, et al. High prevalence of CCDC103 p.His154Pro mutation causing primary ciliary dyskinesia disrupts protein oligomerisation and is associated with normal diagnostic investigations. Thorax 2018; 73: $157-166$.

29 Panizzi JR, Becker-Heck A, Castleman VH, et al. CCDC103 mutations cause primary ciliary dyskinesia by disrupting assembly of ciliary dynein arms. Nat Genet 2012; 44: 714-719.

30 O'Callaghan C, Rutman A, Williams GM, et al. Inner dynein arm defects causing primary ciliary dyskinesia: repeat testing required. Eur Respir J 2011; 38: 603-607.

31 Bukowy-Bieryllo Z, Zietkiewicz E, Loges NT, et al. RPGR mutations might cause reduced orientation of respiratory cilia. Pediatr Pulmonol 2013; 48: 352-363.

32 Jorissen $\mathrm{M}$, Willems $\mathrm{T}$. The secondary nature of ciliary (dis)orientation in secondary and primary ciliary dyskinesia. Acta Otolaryngol 2004; 124: 527-531.

33 Rutman A, Cullinan P, Woodhead M, et al. Ciliary disorientation: a possible variant of primary ciliary dyskinesia. Thorax 1993; 48: 770-771.

34 Boon M, Smits A, Cuppens H, et al. Primary ciliary dyskinesia: critical evaluation of clinical symptoms and diagnosis in patients with normal and abnormal ultrastructure. Orphanet J Rare Dis 2014; 9: 11.

35 Knowles MR, Daniels LA, Davis SD, et al. Primary ciliary dyskinesia. Recent advances in diagnostics, genetics, and characterization of clinical disease. Am J Respir Crit Care Med 2013; 188: 913-922.

36 Kouis P, Yiallouros PK, Middleton N, et al. Prevalence of primary ciliary dyskinesia in consecutive referrals of suspect cases and the transmission electron microscopy detection rate: a systematic review and meta-analysis. Pediatr Res 2017; 81: 398-405.

37 Bartoloni L, Blouin JL, Pan Y, et al. Mutations in the DNAH11 (axonemal heavy chain dynein type 11) gene cause one form of situs inversus totalis and most likely primary ciliary dyskinesia. Proc Natl Acad Sci USA 2002; 99: $10282-10286$. 
38 Knowles MR, Leigh MW, Carson JL, et al. Mutations of DNAH11 in patients with primary ciliary dyskinesia with normal ciliary ultrastructure. Thorax 2012; 67: 433-441.

39 Olbrich H, Schmidts M, Werner C, et al. Recessive HYDIN mutations cause primary ciliary dyskinesia without randomization of left-right body asymmetry. Am J Hum Genet 2012; 91: 672-684.

40 Dixon M, Shoemark A. Secondary defects detected by transmission electron microscopy in primary ciliary dyskinesia diagnostics. Ultrastruct Pathol 2017; 41: 390-398.

41 de Iongh RU, Rutland J. Ciliary defects in healthy subjects, bronchiectasis, and primary ciliary dyskinesia. Am J Respir Crit Care Med 1995; 151: 1559-1567.

42 Rossman CM, Lee RM, Forrest JB, et al. Nasal cilia in normal man, primary ciliary dyskinesia and other respiratory diseases: analysis of motility and ultrastructure. Eur J Respir Dis Suppl 1983; 127: 64-70.

43 Jorissen M, Willems T, Van der Schueren B, et al. Secondary ciliary dyskinesia is absent after ciliogenesis in culture. Acta Otorhinolaryngol Belg 2000; 54: 333-342.

44 Hirst RA, Jackson CL, Coles JL, et al. Culture of primary ciliary dyskinesia epithelial cells at air-liquid interface can alter ciliary phenotype but remains a robust and informative diagnostic aid. PLoS One 2014; 9: e89675. 